\title{
Rapid Quantum Mechanical Models for the Computational Estimation of C-H Bond Dissociation Energies as a Measure of Metabolic Stability
}

John L. Lewin and Christopher J. Cramer*

Department of Chemistry and Supercomputer Institute, University of Minnesota, Minneapolis, MN 55455-0431.

\section{SUPPORTING INFORMATION}

Statistical data for performance of various fitting protocols over the hydrocarbon test set. 
Table S1a. Errors in BDE prediction before and after regression on experimental data for all Morse potential fitting protocols. ${ }^{a}$

\begin{tabular}{|c|c|c|c|c|c|c|c|c|c|c|c|c|c|c|c|c|}
\hline \multirow{3}{*}{$\begin{array}{l}\text { scan type } \\
\text { protocol }\end{array}$} & \multicolumn{8}{|c|}{ AM1 relaxed } & \multicolumn{8}{|c|}{ AM1 unrelaxed } \\
\hline & \multicolumn{3}{|c|}{ before regression } & \multicolumn{5}{|c|}{ after regression } & \multicolumn{3}{|c|}{ before regression } & \multicolumn{5}{|c|}{ after regression } \\
\hline & RMSE & MUE & MSE & slope & intercept & $\mathrm{R}^{2}$ & RMSE & MUE & RMSE & MUE & MSE & slope & intercept & $\mathrm{R}^{2}$ & RMSE & MUE \\
\hline 3.1 & 84.8 & 79.1 & 79.1 & 0.16 & 72.93 & 0.19 & 11.3 & 7.6 & 108.8 & 96.7 & 96.7 & 0.10 & 81.99 & 0.19 & 11.3 & 8.1 \\
\hline 3.2 & 72.4 & 71.7 & 71.7 & 0.60 & -2.44 & 0.77 & 6.0 & 4.9 & 86.2 & 85.8 & 85.8 & 0.66 & -21.28 & 0.64 & 7.5 & 6.3 \\
\hline 3.3 & 72.1 & 71.7 & 71.7 & 0.67 & -13.57 & 0.84 & 5.0 & 4.1 & 85.1 & 85.0 & 85.0 & 0.93 & -72.09 & 0.84 & 5.0 & 4.3 \\
\hline 3.4 & 72.8 & 72.4 & 72.4 & 0.68 & -15.82 & 0.86 & 4.8 & 3.9 & 86.1 & 86.0 & 86.0 & 1.01 & -87.56 & 0.87 & 4.5 & 4.1 \\
\hline 3.5 & 74.2 & 73.9 & 73.9 & 0.68 & -16.96 & 0.86 & 4.6 & 3.8 & 87.5 & 87.4 & 87.4 & 1.03 & -92.78 & 0.88 & 4.4 & 4.0 \\
\hline 3.6 & 74.8 & 74.5 & 74.5 & 0.66 & -15.22 & 0.91 & 3.8 & 3.0 & 89.3 & 89.2 & 89.2 & 1.04 & -96.35 & 0.88 & 4.4 & 3.9 \\
\hline 3.7 & 76.8 & 76.4 & 76.4 & 0.67 & -16.77 & 0.91 & 3.8 & 3.1 & 91.2 & 91.1 & 91.1 & 1.03 & -97.71 & 0.88 & 4.4 & 3.9 \\
\hline 3.8 & 78.8 & 78.5 & 78.5 & 0.66 & -17.68 & 0.91 & 3.8 & 3.2 & 93.1 & 93.0 & 93.0 & 1.03 & -99.50 & 0.88 & 4.4 & 3.8 \\
\hline 5.1 & 72.1 & 71.5 & 71.5 & 0.61 & -4.16 & 0.77 & 6.0 & 4.8 & 86.4 & 85.8 & 85.8 & 0.63 & -17.11 & 0.63 & 7.7 & 6.4 \\
\hline
\end{tabular}

${ }^{a}$ units for RMSE, MUE, and MSE are $\mathrm{kcal} / \mathrm{mol}$; slope, $\mathrm{R}^{2}$ unitless.

\begin{tabular}{|c|c|c|c|c|c|c|c|c|c|c|c|c|c|c|c|c|}
\hline \multirow{3}{*}{$\begin{array}{l}\text { scan type } \\
\text { protocol }\end{array}$} & \multicolumn{8}{|c|}{ PM3 relaxed } & \multicolumn{8}{|c|}{ PM3 unrelaxed } \\
\hline & \multicolumn{3}{|c|}{ before regression } & \multicolumn{5}{|c|}{ after regression } & \multicolumn{3}{|c|}{ before regression } & \multicolumn{5}{|c|}{ after regression } \\
\hline & RMSE & MUE & MSE & slope & intercept & $\mathrm{R}^{2}$ & RMSE & MUE & RMSE & MUE & MSE & slope & intercept & $\mathrm{R}^{2}$ & RMSE & MUE \\
\hline 3.1 & 16.5 & 14.1 & 10.4 & 0.49 & 47.10 & 0.56 & 8.4 & 6.4 & 20.8 & 19.4 & 15.2 & 0.44 & 51.07 & 0.43 & 9.5 & 7.6 \\
\hline 3.2 & 17.1 & 15.1 & 15.1 & 0.70 & 19.89 & 0.71 & 6.7 & 5.7 & 18.8 & 17.4 & 17.4 & 0.78 & 8.54 & 0.73 & 6.6 & 5.4 \\
\hline 3.3 & 17.7 & 16.2 & 16.2 & 0.73 & 15.68 & 0.78 & 5.9 & 5.0 & 20.5 & 19.5 & 19.5 & 0.82 & 1.98 & 0.76 & 6.1 & 5.2 \\
\hline 3.4 & 19.6 & 18.3 & 18.3 & 0.74 & 12.42 & 0.78 & 5.9 & 5.0 & 22.5 & 21.6 & 21.6 & 0.85 & -3.34 & 0.78 & 5.9 & 4.9 \\
\hline 3.5 & 22.0 & 20.9 & 20.9 & 0.76 & 8.81 & 0.78 & 5.8 & 5.0 & 25.2 & 24.5 & 24.5 & 0.87 & -8.36 & 0.79 & 5.8 & 4.9 \\
\hline 3.6 & 25.1 & 24.3 & 24.3 & 0.77 & 4.40 & 0.79 & 5.7 & 4.9 & 28.6 & 28.0 & 28.0 & 0.90 & -14.84 & 0.80 & 5.6 & 4.8 \\
\hline 3.7 & 29.0 & 28.3 & 28.3 & 0.79 & -0.76 & 0.81 & 5.5 & 4.7 & 32.9 & 32.4 & 32.4 & 0.93 & -22.44 & 0.81 & 5.5 & 4.7 \\
\hline 3.8 & 33.7 & 33.1 & 33.1 & 0.80 & -6.42 & 0.82 & 5.3 & 4.5 & 38.0 & 37.6 & 37.6 & 0.95 & -30.81 & 0.82 & 5.3 & 4.6 \\
\hline 5.1 & 17.1 & 15.1 & 15.1 & 0.70 & 20.11 & 0.71 & 6.7 & 5.7 & 18.9 & 17.4 & 17.4 & 0.78 & 8.87 & 0.72 & 6.6 & 5.4 \\
\hline
\end{tabular}


Table S1a. Continued.

\begin{tabular}{|c|c|c|c|c|c|c|c|c|c|c|c|c|c|c|c|c|}
\hline \multirow{3}{*}{$\begin{array}{l}\text { scan type } \\
\text { protocol }\end{array}$} & \multicolumn{8}{|c|}{ HF/MIDI relaxed } & \multicolumn{8}{|c|}{ HF/MIDI unrelaxed } \\
\hline & \multicolumn{3}{|c|}{ before regression } & \multicolumn{5}{|c|}{ after regression } & \multicolumn{3}{|c|}{ before regression } & \multicolumn{5}{|c|}{ after regression } \\
\hline & RMSE & MUE & MSE & slope & intercept & $\mathrm{R}^{2}$ & RMSE & MUE & RMSE & MUE & MSE & slope & intercept & $\mathrm{R}^{2}$ & RMSE & MUE \\
\hline 3.1 & 18.3 & 11.4 & -4.1 & 0.34 & 68.40 & 0.39 & 9.9 & 7.6 & 10.5 & 8.1 & 2.3 & 0.65 & 34.71 & 0.47 & 9.1 & 6.7 \\
\hline 3.2 & 7.8 & 5.4 & -1.2 & 0.70 & 31.04 & 0.76 & 6.2 & 4.1 & 7.9 & 6.1 & 5.3 & 0.99 & -4.51 & 0.78 & 5.9 & 4.5 \\
\hline 3.3 & 5.2 & 4.1 & 0.4 & 0.84 & 15.96 & 0.86 & 4.7 & 3.1 & 8.0 & 6.1 & 6.1 & 1.05 & -12.02 & 0.83 & 5.2 & 4.4 \\
\hline 3.4 & 4.6 & 3.4 & 1.5 & 0.90 & 9.27 & 0.89 & 4.1 & 2.8 & 8.5 & 6.8 & 6.8 & 1.08 & -15.25 & 0.84 & 5.0 & 4.2 \\
\hline 3.5 & 4.7 & 3.3 & 2.4 & 0.92 & 5.92 & 0.90 & 3.9 & 2.8 & 9.1 & 7.7 & 7.7 & 1.09 & -17.66 & 0.85 & 4.9 & 4.2 \\
\hline 3.6 & 5.1 & 3.8 & 3.3 & 0.93 & 3.60 & 0.91 & 3.8 & 2.7 & 9.9 & 8.5 & 8.5 & 1.10 & -19.72 & 0.85 & 4.8 & 4.1 \\
\hline 3.7 & 5.7 & 4.4 & 4.2 & 0.94 & 1.89 & 0.91 & 3.8 & 2.8 & 10.7 & 9.5 & 9.5 & 1.11 & -21.85 & 0.85 & 4.8 & 4.1 \\
\hline 3.8 & 6.4 & 5.2 & 5.2 & 0.95 & 0.38 & 0.91 & 3.7 & 2.9 & 11.6 & 10.5 & 10.5 & 1.12 & -24.00 & 0.86 & 4.8 & 4.1 \\
\hline 5.1 & 8.1 & 5.6 & -1.4 & 0.69 & 32.64 & 0.75 & 6.3 & 4.3 & 7.8 & 5.9 & 5.1 & 1.03 & -8.19 & 0.78 & 5.9 & 4.7 \\
\hline
\end{tabular}

\begin{tabular}{|c|c|c|c|c|c|c|c|c|c|c|c|c|c|c|c|c|}
\hline \multirow{3}{*}{$\begin{array}{l}\text { scan type } \\
\text { protocol }\end{array}$} & \multicolumn{8}{|c|}{ B3LYP/MIDI relaxed } & \multicolumn{8}{|c|}{ B3LYP/MIDI unrelaxed } \\
\hline & \multicolumn{3}{|c|}{ before regression } & \multicolumn{5}{|c|}{ after regression } & \multicolumn{3}{|c|}{ before regression } & \multicolumn{5}{|c|}{ after regression } \\
\hline & RMSE & MUE & MSE & slope & intercept & $\mathrm{R}^{2}$ & RMSE & MUE & RMSE & MUE & MSE & slope & intercept & $\mathrm{R}^{2}$ & RMSE & MUE \\
\hline 3.1 & 26.2 & 17.7 & -17.1 & 0.20 & 85.34 & 0.10 & 11.9 & 8.2 & 10.0 & 8.8 & -6.6 & 0.92 & 14.05 & 0.64 & 7.5 & 6.0 \\
\hline 3.2 & 16.3 & 14.4 & -14.4 & 0.81 & 30.86 & 0.66 & 7.4 & 4.9 & 8.4 & 7.4 & -6.9 & 1.24 & -15.56 & 0.89 & 4.1 & 3.7 \\
\hline 3.3 & 13.7 & 12.8 & -12.8 & 1.04 & 9.07 & 0.86 & 4.7 & 3.1 & 8.3 & 7.5 & -6.8 & 1.28 & -19.59 & 0.89 & 4.1 & 3.7 \\
\hline 3.4 & 12.5 & 11.9 & -11.9 & 1.10 & 2.51 & 0.91 & 3.7 & 2.4 & 7.9 & 7.1 & -6.3 & 1.29 & -21.22 & 0.90 & 4.0 & 3.6 \\
\hline 3.5 & 11.7 & 11.1 & -11.1 & 1.13 & -0.69 & 0.93 & 3.3 & 2.3 & 7.5 & 6.8 & -5.8 & 1.30 & -23.27 & 0.90 & 4.0 & 3.6 \\
\hline 3.6 & 10.9 & 10.4 & -10.4 & 1.14 & -2.72 & 0.94 & 3.1 & 2.3 & 7.1 & 6.3 & -5.1 & 1.31 & -25.11 & 0.90 & 3.9 & 3.5 \\
\hline 3.7 & 10.3 & 9.7 & -9.7 & 1.15 & -4.44 & 0.94 & 3.1 & 2.2 & 6.6 & 5.8 & -4.4 & 1.32 & -27.18 & 0.90 & 3.9 & 3.5 \\
\hline 3.8 & 9.6 & 9.0 & -9.0 & 1.16 & -6.13 & 0.94 & 3.0 & 2.2 & 6.1 & 5.5 & -3.7 & 1.33 & -29.05 & 0.90 & 3.9 & 3.5 \\
\hline 5.1 & 16.6 & 14.5 & -14.5 & 0.79 & 33.17 & 0.63 & 7.6 & 5.1 & 8.4 & 7.5 & -7.0 & 1.24 & -15.68 & 0.89 & 4.1 & 3.7 \\
\hline
\end{tabular}


Table S1b. Errors in BDE prediction after regression on experimental data for all 3-point parabola fitting protocols. $^{a}$

\begin{tabular}{|c|c|c|c|c|c|c|c|c|c|c|}
\hline \multirow{2}{*}{$\begin{array}{l}\text { scan type } \\
\text { protocol }\end{array}$} & \multicolumn{5}{|c|}{ AM1 relaxed } & \multicolumn{5}{|c|}{ AM1 unrelaxed } \\
\hline & slope & intercept & $\mathrm{R}^{2}$ & RMSE & MUE & slope & intercept & $\mathrm{R}^{2}$ & RMSE & MUE \\
\hline 3.1 & 0.18 & -34.3 & 0.68 & 7.1 & 4.7 & 0.24 & -82.38 & 0.84 & 5.0 & 4.3 \\
\hline 3.2 & 0.20 & -54.0 & 0.84 & 5.1 & 4.3 & 0.23 & -83.15 & 0.84 & 5.1 & 4.4 \\
\hline 3.3 & 0.20 & -55.3 & 0.85 & 4.9 & 4.2 & 0.23 & -83.84 & 0.84 & 5.1 & 4.4 \\
\hline 3.4 & 0.20 & -56.9 & 0.85 & 4.9 & 4.1 & 0.23 & -85.55 & 0.84 & 5.1 & 4.4 \\
\hline 3.5 & 0.20 & -59.3 & 0.85 & 4.8 & 4.1 & 0.23 & -87.58 & 0.84 & 5.1 & 4.4 \\
\hline 3.6 & 0.19 & -62.3 & 0.86 & 4.7 & 4.0 & 0.22 & -90.00 & 0.84 & 5.1 & 4.4 \\
\hline 3.7 & 0.19 & -65.5 & 0.86 & 4.7 & 4.0 & 0.22 & -92.43 & 0.84 & 5.1 & 4.3 \\
\hline 3.8 & 0.19 & -68.8 & 0.86 & 4.7 & 4.0 & 0.21 & -95.10 & 0.84 & 5.1 & 4.3 \\
\hline
\end{tabular}

${ }^{a}$ units for RMSE, MUE, and MSE are $\mathrm{kcal} / \mathrm{mol}$; slope, $\AA^{2} ; \mathrm{R}^{2}$, unitless.

\begin{tabular}{|c|c|c|c|c|c|c|c|c|c|c|}
\hline \multirow{2}{*}{$\begin{array}{l}\text { scan type } \\
\text { protocol }\end{array}$} & \multicolumn{5}{|c|}{ PM3 relaxed } & \multicolumn{5}{|c|}{ PM3 unrelaxed } \\
\hline & slope & intercept & $\mathrm{R}^{2}$ & RMSE & MUE & slope & intercept & $\mathrm{R}^{2}$ & RMSE & MUE \\
\hline 3.1 & 0.16 & -17.0 & 0.48 & 9.0 & 7.9 & 0.13 & 0.07 & 0.34 & 10.2 & 8.6 \\
\hline 3.2 & 0.16 & -19.1 & 0.48 & 9.0 & 7.9 & 0.14 & -4.22 & 0.35 & 10.1 & 8.6 \\
\hline 3.3 & 0.16 & -23.5 & 0.49 & 8.9 & 7.8 & 0.14 & -7.07 & 0.35 & 10.1 & 8.6 \\
\hline 3.4 & 0.17 & -27.5 & 0.49 & 8.9 & 7.8 & 0.14 & -11.18 & 0.36 & 10.1 & 8.5 \\
\hline 3.5 & 0.17 & -32.5 & 0.49 & 8.9 & 7.8 & 0.14 & -16.57 & 0.36 & 10.0 & 8.5 \\
\hline 3.6 & 0.17 & -38.6 & 0.50 & 8.9 & 7.8 & 0.15 & -23.20 & 0.37 & 10.0 & 8.5 \\
\hline 3.7 & 0.17 & -45.4 & 0.50 & 8.9 & 7.8 & 0.15 & -31.04 & 0.38 & 9.9 & 8.4 \\
\hline 3.8 & 0.17 & -52.7 & 0.51 & 8.8 & 7.7 & 0.15 & -39.92 & 0.40 & 9.7 & 8.3 \\
\hline
\end{tabular}

\begin{tabular}{|c|c|c|c|c|c|c|c|c|c|c|}
\hline \multirow{2}{*}{$\begin{array}{l}\text { scan type } \\
\text { protocol }\end{array}$} & \multicolumn{5}{|c|}{ HF/MIDI relaxed } & \multicolumn{5}{|c|}{ HF/MIDI unrelaxed } \\
\hline & slope & intercept & $\mathrm{R}^{2}$ & RMSE & $\overline{\text { MUE }}$ & slope & intercept & $\mathrm{R}^{2}$ & RMSE & $\overline{\text { MUE }}$ \\
\hline 3.1 & 0.18 & -51.6 & 0.90 & 4.0 & 3.0 & 0.20 & -71.82 & 0.86 & 4.7 & 4.0 \\
\hline 3.2 & 0.19 & -61.3 & 0.90 & 4.0 & 3.2 & 0.20 & -72.14 & 0.86 & 4.7 & 4.0 \\
\hline 3.3 & 0.19 & -64.3 & 0.89 & 4.1 & 3.4 & 0.20 & -73.88 & 0.86 & 4.7 & 4.0 \\
\hline 3.4 & 0.18 & -67.2 & 0.89 & 4.2 & 3.5 & 0.19 & -75.93 & 0.86 & 4.7 & 4.0 \\
\hline 3.5 & 0.17 & -70.1 & 0.88 & 4.3 & 3.6 & 0.18 & -78.24 & 0.86 & 4.7 & 4.0 \\
\hline 3.6 & 0.17 & -73.1 & 0.88 & 4.4 & 3.7 & 0.17 & -80.54 & 0.86 & 4.7 & 4.0 \\
\hline 3.7 & 0.16 & -75.9 & 0.88 & 4.4 & 3.7 & 0.16 & -82.81 & 0.86 & 4.7 & 4.0 \\
\hline 3.8 & 0.14 & -78.7 & 0.87 & 4.5 & 3.8 & 0.15 & -84.94 & 0.86 & 4.7 & 4.0 \\
\hline
\end{tabular}

\begin{tabular}{|c|c|c|c|c|c|c|c|c|c|c|}
\hline \multirow{2}{*}{$\begin{array}{l}\text { scan type } \\
\text { protocol }\end{array}$} & \multicolumn{5}{|c|}{ B3LYP/MIDI relaxed } & \multicolumn{5}{|c|}{ B3LYP/MIDI unrelaxed } \\
\hline & slope & intercept & $\mathrm{R}^{2}$ & RMSE & MUE & slope & intercept & $\mathrm{R}^{2}$ & RMSE & MUE \\
\hline 3.1 & 0.20 & -47.9 & 0.91 & 3.7 & 2.9 & 0.22 & -66.54 & 0.87 & 4.5 & 4.0 \\
\hline 3.2 & 0.20 & -53.2 & 0.91 & 3.8 & 3.2 & 0.21 & -64.72 & 0.86 & 4.7 & 4.1 \\
\hline 3.3 & 0.20 & -56.0 & 0.90 & 4.0 & 3.5 & 0.21 & -66.03 & 0.86 & 4.7 & 4.1 \\
\hline 3.4 & 0.19 & -58.4 & 0.89 & 4.1 & 3.6 & 0.20 & -67.83 & 0.86 & 4.7 & 4.1 \\
\hline 3.5 & 0.18 & -61.1 & 0.89 & 4.2 & 3.7 & 0.19 & -70.04 & 0.86 & 4.7 & 4.1 \\
\hline 3.6 & 0.17 & -63.8 & 0.88 & 4.3 & 3.7 & 0.18 & -72.19 & 0.86 & 4.7 & 4.1 \\
\hline 3.7 & 0.16 & -66.4 & 0.88 & 4.3 & 3.8 & 0.17 & -74.34 & 0.86 & 4.7 & 4.1 \\
\hline 3.8 & 0.15 & -69.0 & 0.88 & 4.4 & 3.8 & 0.16 & -76.26 & 0.86 & 4.7 & 4.1 \\
\hline
\end{tabular}


Table S1c. Errors in BDE prediction after regression on experimental data for all 2-point parabola fitting protocols. $^{a}$

\begin{tabular}{|c|c|c|c|c|c|c|c|c|c|c|}
\hline \multirow{2}{*}{$\begin{array}{l}\text { scan type } \\
\text { protocol }\end{array}$} & \multicolumn{5}{|c|}{ AM1 relaxed } & \multicolumn{5}{|c|}{ AM1 unrelaxed } \\
\hline & slope & intercept & $\mathrm{R}^{2}$ & RMSE & $\overline{M U E}$ & slope & intercept & $\mathrm{R}^{2}$ & RMSE & MUE \\
\hline 3.1 & 0.20 & -37.7 & 0.71 & 6.8 & 4.7 & 0.25 & -81.4 & 0.86 & 4.7 & 3.9 \\
\hline 3.2 & 0.23 & -50.5 & 0.84 & 5.0 & 4.2 & 0.28 & -84.4 & 0.85 & 4.9 & 4.1 \\
\hline 3.3 & 0.25 & -49.6 & 0.85 & 4.8 & 4.1 & 0.30 & -85.2 & 0.85 & 4.9 & 4.2 \\
\hline 3.4 & 0.26 & -48.7 & 0.86 & 4.7 & 4.0 & 0.32 & -87.8 & 0.85 & 4.9 & 4.2 \\
\hline 3.5 & 0.28 & -48.2 & 0.86 & 4.7 & 3.9 & 0.35 & -90.1 & 0.85 & 4.8 & 4.2 \\
\hline 3.6 & 0.30 & -47.6 & 0.89 & 4.2 & 3.4 & 0.38 & -92.9 & 0.85 & 4.8 & 4.2 \\
\hline 3.7 & 0.32 & -47.5 & 0.89 & 4.1 & 3.3 & 0.41 & -95.2 & 0.85 & 4.8 & 4.2 \\
\hline 3.8 & 0.34 & -47.2 & 0.89 & 4.1 & 3.3 & 0.44 & -97.7 & 0.86 & 4.8 & 4.1 \\
\hline
\end{tabular}

$a$ units for RMSE, MUE, and MSE are $\mathrm{kcal} / \mathrm{mol}$; slope, $\AA^{2} ; \mathrm{R}^{2}$, unitless.

\begin{tabular}{|c|c|c|c|c|c|c|c|c|c|c|}
\hline \multirow{2}{*}{$\begin{array}{l}\text { scan type } \\
\text { protocol }\end{array}$} & \multicolumn{5}{|c|}{ PM3 relaxed } & \multicolumn{5}{|c|}{ PM3 unrelaxed } \\
\hline & slope & intercept & $\mathrm{R}^{2}$ & RMSE & MUE & slope & intercept & $\mathrm{R}^{2}$ & RMSE & MUE \\
\hline 3.1 & 0.19 & -23.1 & 0.54 & 8.5 & 7.5 & 0.17 & -13.6 & 0.41 & 9.7 & 8.3 \\
\hline 3.2 & 0.20 & -20.5 & 0.54 & 8.5 & 7.5 & 0.19 & -15.9 & 0.44 & 9.4 & 8.1 \\
\hline 3.3 & 0.22 & -22.6 & 0.59 & 8.1 & 7.2 & 0.21 & -20.1 & 0.48 & 9.1 & 7.9 \\
\hline 3.4 & 0.24 & -24.2 & 0.61 & 7.9 & 7.0 & 0.24 & -26.6 & 0.52 & 8.7 & 7.6 \\
\hline 3.5 & 0.27 & -27.0 & 0.63 & 7.6 & 6.8 & 0.28 & -34.4 & 0.56 & 8.3 & 7.3 \\
\hline 3.6 & 0.30 & -31.1 & 0.66 & 7.3 & 6.5 & 0.32 & -43.3 & 0.61 & 7.9 & 6.9 \\
\hline 3.7 & 0.33 & -36.1 & 0.69 & 7.0 & 6.2 & 0.37 & -53.1 & 0.65 & 7.5 & 6.5 \\
\hline 3.8 & 0.37 & -41.6 & 0.72 & 6.6 & 5.9 & 0.42 & -63.4 & 0.69 & 7.0 & 6.0 \\
\hline
\end{tabular}

\begin{tabular}{|c|c|c|c|c|c|c|c|c|c|c|}
\hline \multirow{2}{*}{$\begin{array}{l}\text { scan type } \\
\text { protocol }\end{array}$} & \multicolumn{5}{|c|}{ HF/MIDI relaxed } & \multicolumn{5}{|c|}{ HF/MIDI unrelaxed } \\
\hline & slope & intercept & $\mathrm{R}^{2}$ & RMSE & $\overline{\text { MUE }}$ & slope & intercept & $\mathrm{R}^{2}$ & RMSE & $\overline{\text { MUE }}$ \\
\hline 3.1 & 0.18 & -31.3 & 0.84 & 5.0 & 3.4 & 0.22 & -64.1 & 0.84 & 5.0 & 4.2 \\
\hline 3.2 & 0.21 & -43.4 & 0.89 & 4.1 & 3.0 & 0.23 & -61.4 & 0.85 & 4.9 & 4.1 \\
\hline 3.3 & 0.23 & -42.6 & 0.90 & 4.0 & 2.9 & 0.25 & -58.7 & 0.85 & 4.8 & 4.1 \\
\hline 3.4 & 0.25 & -40.1 & 0.90 & 3.9 & 3.0 & 0.27 & -56.1 & 0.86 & 4.8 & 4.1 \\
\hline 3.5 & 0.26 & -37.0 & 0.90 & 3.9 & 3.1 & 0.29 & -53.9 & 0.86 & 4.8 & 4.1 \\
\hline 3.6 & 0.28 & -34.1 & 0.90 & 3.9 & 3.1 & 0.31 & -51.9 & 0.86 & 4.7 & 4.1 \\
\hline 3.7 & 0.30 & -31.4 & 0.90 & 3.9 & 3.1 & 0.33 & -50.3 & 0.86 & 4.7 & 4.1 \\
\hline 3.8 & 0.32 & -29.0 & 0.90 & 3.9 & 3.1 & 0.36 & -49.1 & 0.86 & 4.7 & 4.1 \\
\hline
\end{tabular}

\begin{tabular}{|c|c|c|c|c|c|c|c|c|c|c|}
\hline \multirow{2}{*}{$\begin{array}{l}\text { scan type } \\
\text { protocol }\end{array}$} & \multicolumn{5}{|c|}{ B3LYP/MIDI relaxed } & \multicolumn{5}{|c|}{ B3LYP/MIDI unrelaxed } \\
\hline & slope & intercept & $\mathrm{R}^{2}$ & RMSE & MUE & slope & intercept & $\mathrm{R}^{2}$ & RMSE & MUE \\
\hline$\overline{3.1}$ & 0.20 & -30.8 & 0.83 & 5.1 & 3.2 & 0.24 & -63.0 & 0.87 & 4.6 & 4.0 \\
\hline 3.2 & 0.24 & -42.5 & 0.91 & 3.7 & 2.7 & 0.25 & -57.3 & 0.86 & 4.6 & 4.0 \\
\hline 3.3 & 0.26 & -42.2 & 0.92 & 3.5 & 2.7 & 0.27 & -54.6 & 0.86 & 4.8 & 4.1 \\
\hline 3.4 & 0.28 & -39.5 & 0.92 & 3.5 & 2.7 & 0.29 & -52.2 & 0.86 & 4.7 & 4.1 \\
\hline 3.5 & 0.30 & -37.1 & 0.92 & 3.5 & 2.8 & 0.32 & -50.5 & 0.86 & 4.8 & 4.1 \\
\hline 3.6 & 0.32 & -34.8 & 0.93 & 3.4 & 2.8 & 0.34 & -48.9 & 0.86 & 4.7 & 4.1 \\
\hline 3.7 & 0.34 & -32.8 & 0.93 & 3.4 & 2.8 & 0.37 & -47.8 & 0.86 & 4.8 & 4.1 \\
\hline 3.8 & 0.36 & -31.2 & 0.93 & 3.3 & 2.7 & 0.39 & -46.8 & 0.86 & 4.7 & 4.1 \\
\hline
\end{tabular}

